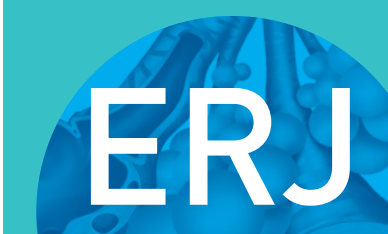

open research
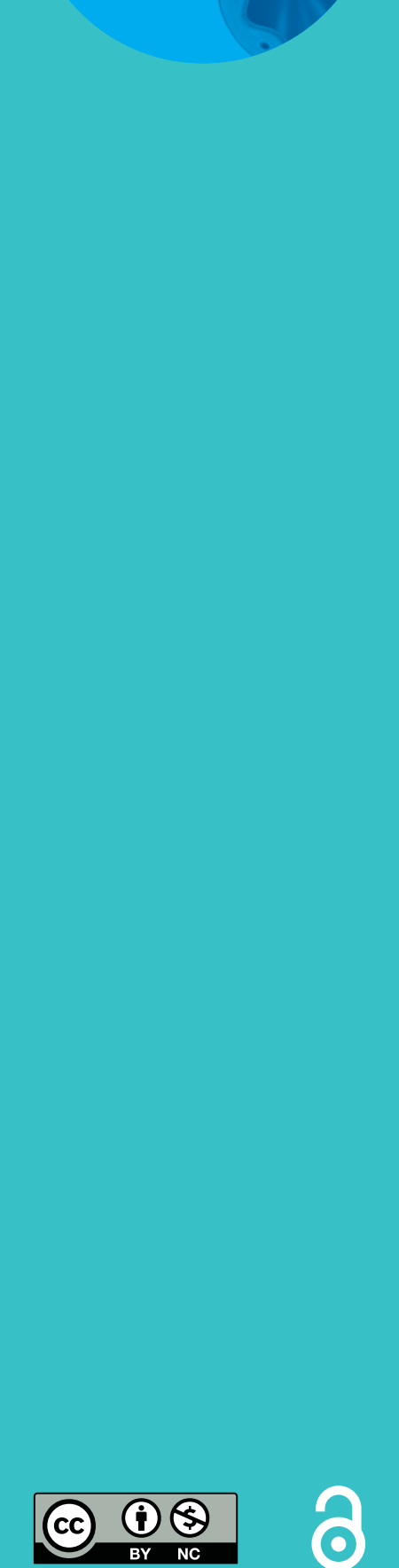

\section{Pulmonary tularaemia: a differential diagnosis to lung cancer}

\author{
Astrid Kravdal ${ }^{1}, \varnothing y s t e i n$ Olav Stubhaug ${ }^{1}$, Anne Grete Wåg $\varnothing^{2}$, \\ Magnus Steien Sætereng ${ }^{3}$, Dag Amundsen ${ }^{4}$, Ruta Piekuviene ${ }^{5}$ and \\ Annette Kristiansen ${ }^{6}$
}

Affiliations: ${ }^{1}$ Dept of Medicine, Innlandet Hospital Trust, Gjøvik, Norway. ${ }^{2}$ Dept of Microbiology, St Olav's Hospital, Trondheim University Hospital, Trondheim, Norway. ${ }^{3}$ Dept of Medicine, Innlandet Hospital Trust, Elverum, Norway. ${ }^{4}$ Dept of Medicine, Innlandet Hospital Trust, Lillehammer, Norway. ${ }^{5}$ Dept of Radiology, Innlandet Hospital Trust, Gjøvik, Norway. ${ }^{6}$ Dept of Pulmonary Medicine, Oslo University Hospital, Ullevål, Norway.

Correspondence: Astrid Kravdal, Dept of Medicine, Innlandet Hospital Trust, N-2819 Gjøvik, Norway. E-mail: astrid.kravdaldsykehuset-innlandet.no

\section{ABSTRACT}

Background: Pulmonary manifestations of tularaemia are reported to be infrequent in previous publications. During 2016, we noticed an increase in the number of hospitalised patients with pulmonary tularaemia in Eastern Norway. We aimed to investigate primary pulmonary tularaemia in Eastern Norway in terms of symptoms, radiological and microbiological findings, incidence and risk exposure.

Methods: A retrospective analysis of consecutive primary pulmonary tularaemia cases from 2016 until 2018 was conducted.

Results: From 1 September, 2016 until 31 December, 2018, 22 patients were diagnosed with primary pulmonary tularaemia at Innlandet Hospital Trust, representing $48 \%$ of all reported tularaemia cases in the region. A peak annual incidence of 3.4 in 100000 was found in 2017.

All 22 patients lived in, or had recently visited, rural areas. Eighteen patients reported risk exposure for tularaemia, such as wood chopping, outdoor activities and farming prior to symptom onset. All patients experienced fever, and 19 patients had respiratory symptoms. Ten patients were in spontaneous recovery at diagnosis.

All patients had a chest computed tomography scan. Overall, 19 patients had pulmonary lesions and 18 had enlarged intrathoracic lymph nodes. The computed tomography images were described as suspicious of malignancy in 17 patients.

Conclusion: Pulmonary manifestations in tularaemia occurred more frequently in our region than expected from previous reports. Although all patients had symptoms consistent with infection, the majority were primarily investigated considering lung cancer due to the radiology report. In endemic areas, pulmonary tularaemia may be an important differential diagnosis to lung cancer.

@ERSpublications

Pulmonary tularaemia is possibly more frequent than previously reported. Due to similar radiological findings, pulmonary tularaemia may be an important differential diagnosis to lung cancer in endemic areas. https://bit.ly/2RcEPfN

Cite this article as: Kravdal A, Stubhaug ØO, Wågø AG, et al. Pulmonary tularaemia: a differential diagnosis to lung cancer. ERJ Open Res 2020; 6: 00093-2019 [https://doi.org/10.1183/ 23120541.00093-2019].

This article has supplementary material available from openres.ersjournals.com

Received: 11 April 2019 | Accepted after revision: 24 March 2020

Copyright $\odot$ ERS 2020. This article is open access and distributed under the terms of the Creative Commons Attribution Non-Commercial Licence 4.0. 


\section{Introduction}

Tularaemia is a rare zoonosis, caused by Francisella tularensis, a small intracellular Gram-negative bacterium. The species is separated into three subspecies, two of which are of clinical importance. Infections caused by F. tularensis subspecies tularensis occur only in North America. F. tularensis subsp. holarctica occurs in North America, most of the countries in Europe and in Asia, from Russia to China and Japan, and is generally associated with a less aggressive disease than the subspecies tularensis [1-4]. Sweden, Finland, Norway, Slovakia, Spain, Turkey and Hungary have the highest reported incidence worldwide [5]. Tularaemia has only sporadically been identified on the southern hemisphere [4, 6, 7]. In Norway, notification of tularaemia cases is mandatory by law. The reported incidence in Norway has increased in the last two decades $[2,5,8]$.

Tularaemia has six major clinical manifestations according to the clinical picture and site of inoculation: ulceroglandular, glandular, oculoglandular, oropharyngeal, pulmonary and typhoidal [3, 4]. In Europe, ulceroglandular manifestations predominate, whereas pulmonary manifestations of tularaemia are reported to be uncommon. Water-born oropharyngeal forms are the most frequent in Norway, whereas in the neighbouring countries of Sweden and Finland, glandular and ulceroglandular manifestations transmitted by insect bites are more common $[2,8,9]$. Pulmonary tularaemia usually results from inhalation of $F$. tularensis, but can occur as secondary pulmonary tularaemia through haematogenous spread. Several activities, such as farming and hunting, may result in aerosolisation of the bacteria. The infective dose in humans is extremely low [3, 10-12].

Pulmonary tularaemia classically presents with a flu-like illness with fever, headache, fatigue, joint pain, nausea or diarrhoea, often with respiratory symptoms as cough, dyspnoea and chest pain $[3,4,13]$. There are few recent studies on radiological findings in pulmonary tularaemia with older studies typically describing infiltrates and hilar adenopathy on chest radiography [14-16]. In 2017, VÄRYNEN et. al [13] found enlarged hilar and/or mediastinal lymph nodes to be the most common finding on computed tomography (CT) scan of the chest. The World Health Organization (WHO) guideline states that the diagnosis of tularaemia should be based on serological tests, molecular detection or culture [4].

During 2016, we began noticing an apparent increase in hospitalised patients with pulmonary tularaemia in Eastern Norway. The aim of this retrospective study was to investigate primary pulmonary tularaemia in Eastern Norway, in terms of symptoms, radiological and microbiological findings, incidence and epidemiological risk factors.

\section{Materials and methods}

\section{Methods}

This was an observational study with a retrospective analysis of consecutive pulmonary tularaemia cases diagnosed at Innlandet Hospital Trust from 1 September, 2016 until 31 December, 2018.

Baseline characteristics on epidemiology, presumed mode of transmission, clinical course, initial radiological findings, laboratory results, supplementary investigations and results of follow-up chest radiology were recorded.

Data from the electronic patient records, from the national registry of the Norwegian Surveillance System for Communicable Diseases, and from the National reference laboratory for tularaemia were collected. In cases of missing data, medical records from primary care were examined or follow-up patient interviews were performed as needed.

The Regional Committee for Ethics in Medical Research approved the study (REK 2017/2523), and all patients provided written informed consent.

\section{Microbiological analyses}

The microbiological specimens were analysed by the National Reference Laboratory for Tularaemia at St. Olavs Hospital, Trondheim. The analyses included serological tests, PCR and culture. An in-house microagglutination (MA) test and a commercial IgM and IgG ELISA test (Serion ELISA classic $F$. tularensis IgG/IgM, Institute Virion/Serion $\mathrm{GmbH}$, Würzburg, Germany) were used for the serological diagnosis of tularaemia. The MA test used formalin-killed F. tularensis isolated from a dead hare. A real-time TaqMan PCR targeting the tul4 gene was used for detection of $F$. tularensis directly in clinical specimens [9, 17]. Culture was performed using standard bacteriology medium, including cystine heart agar with and without antibiotics and incubated in $5 \% \mathrm{CO}_{2}$ at $35^{\circ} \mathrm{C}$ for up to 10 days.

\section{Definitions and inclusion criteria}

The diagnosis was based on the combination of a positive microbiological test, according to the WHO guidelines [4], and radiological findings consistent with pulmonary tularaemia [13]. 
Radiological findings on chest CT scan consistent with pulmonary tularaemia, were defined as either

1) Enlarged (>1 cm in short-axis diameter) mediastinal or hilar lymph nodes and/or

2) Pulmonary lesions

A positive microbiological test was defined as fulfilling one or more of the following criteria:

1) Positive F. tularensis culture.

2) Positive F. tularensis PCR.

3) Positive serology:

a) Seroconversion or a fourfold or greater rise in titres of specific antibodies in paired serum samples and/or

b) An MA titre $\geqslant 64$ and positive specific IgM and IgG ELISA.

A potential risk exposure to tularaemia was defined as activities performed during the previous 3 months that could aerosolise the bacterium.

\section{Exclusion criteria}

Patients diagnosed with secondary pulmonary tularaemia, defined as having typical radiological chest findings combined with other organ manifestations of tularaemia, were excluded.

\section{Statistical analyses}

Data were stored and later processed in Microsoft Excel. Continuous variables were described as median and range and categorical variables as proportions or percentages.

\section{Results}

A total of 22 patients with primary pulmonary tularaemia were diagnosed at Innlandet Hospital Trust from 1 September, 2016 until 31 December, 2018. Innlandet Hospital Trust consists of six small and medium-sized hospitals in Hedmark and Oppland counties in Eastern Norway, with a total of 380000 inhabitants. Retrospectively, we performed searches for additional cases in the period 2016-2018 in the patient record system, using the ICD 10 code A21.2, pulmonary tularaemia. We did not identify any patients diagnosed with pulmonary tularaemia at Innlandet Hospital Trust from 1 January, until 31 August, 2016. A peak annual incidence of 3.4 in 100000 was found in 2017. Primary pulmonary tularaemia represented $48 \%$ of all reported tularaemia cases between 2016 and 2018 in this region. Patient characteristics are presented in table 1 , and incidence in table 2.

All patients lived in, or had visited, rural areas during the previous 3 months. A potential risk exposure to tularaemia was reported by 18 patients. Five patients had several possible routes of transmission. Most common routes were wood chopping (five patients), farming (five patients), carpentry/house rehabilitation (three patients), hunting (two patients) and other outdoor activities (six patients). All but one experienced symptom onset in the autumn (figure 1).

\section{Clinical course}

All patients had a fever, and 14 patients were referred to secondary care due to suspicion of infection. Respiratory symptoms such as cough, chest pain or dyspnoea were reported by 19 patients (figure 2). Ten patients were in spontaneous recovery at the time of diagnosis. Overall, 20 patients were treated with

TABLE 1 Patient characteristics of 22 patients diagnosed with primary pulmonary tularaemia

at Innlandet Hospital Trust in Norway from 1 September, 2016 to 31 December, 2018

$\begin{array}{lc}\text { Female } & 6 / 22 \\ \text { Male } & 16 / 22 \\ \text { Age years } & 58.5(38-78] \\ \text { Living in/visiting rural areas during the last 3 months } & 22 / 22 \\ \text { Never smoked } & 8 / 22 \\ \text { Former smoker } & 8 / 22 \\ \text { Current smoker } & 6 / 22 \\ \text { Investigated in fast-track cancer programmes during the course } & 15 / 22 \\ \text { Referred to secondary care due to symptoms of infection } & 14 / 22 \\ \text { Referred to secondary care directly under fast-track cancer programs } & 8 / 22 \\ \text { Duration from referral to diagnosis days } & 17.5(7-75) \\ >\mathbf{3} \text { weeks from referral to diagnosis } & 10 / 22\end{array}$

Data are presented as $\mathrm{n} / \mathrm{N}$ or median (range). 
TABLE 2 Epidemiology of primary pulmonary tularaemia in Hedmark and Oppland counties in Eastern Norway, in 2016-2018

\begin{tabular}{|c|c|c|c|c|}
\hline & 2016 & 2017 & 2018 & 2016-2018 \\
\hline Tularaemia cases in our region" & 8 & 25 & 13 & 46 \\
\hline The incidence of pulmonary tularaemia in our region & $1.1 / 100000$ & $3.4 / 100000$ & $1.3 / 100000$ & $1.9 / 100000$ \\
\hline The incidence of tularaemia in our region & $2.1 / 100000$ & $6.6 / 100000$ & $3.4 / 100000$ & $4.0 / 100000$ \\
\hline The incidence of tularaemia in Norway" & $0.8 / 100000$ & $1.7 / 100000$ & $1.1 / 100000$ & $1.2 / 100000$ \\
\hline
\end{tabular}

ciprofloxacin, for a median of 14 days (range 5-21 days). None of the patients were immunosuppressed, and all experienced a full recovery.

\section{Laboratory results}

In all patients, the values of serum C-reactive protein (CRP) analysed in primary or secondary care were elevated, with a peak median value of $155 \mathrm{mg} \cdot \mathrm{L}^{-1}$ (range $37-259$, normal value $<5$ ).

All patients had positive serological tests. In five, the initial test was negative with a subsequent control test (performed at a median of 17 days after the first test (range 10-51 days)) revealing seroconversion in all five. These patients experienced symptom onset 7-12 days prior to testing, while the rest had symptom onset more than 3 weeks (range 21 days - 5 months) before testing. A total of 329 serological tularaemia tests were ordered in Hedmark and Oppland counties from 1 September, 2016 until 31 December, 2018.

Samples from 11 patients were investigated using PCR analysis and culture (table 3). Four had a positive PCR test. None of the patients had a positive culture for F. tularensis.

\section{Investigations}

All patients were examined with a chest CT scan, and 10 underwent a supplementary fluorodeoxyglucose (FDG) positron emission tomography (PET). Bronchoscopy was performed in 15 patients. Normal endobronchial findings were described in eight patients, signs of inflammation in six patients (from minimal to widespread with necrosis), and one patient had bronchial narrowing and mucosal changes strongly suspicious of malignancy. Four patients underwent a CT-guided lung biopsy, twice in one patient. A total of 10 patients were referred to supplementary endobronchial ultrasound (EBUS), five of which were performed. Serological tests were not available for any of the patients prior to performing supplementary investigations such as bronchoscopy, EBUS and CT-guided biopsy. Investigations are presented in table 4 .

FIGURE 1 The month of symptom onset in 22 patients diagnosed with primary pulmonary tularaemia in Eastern Norway from 1 September, 2016 until 31 December, 2018.

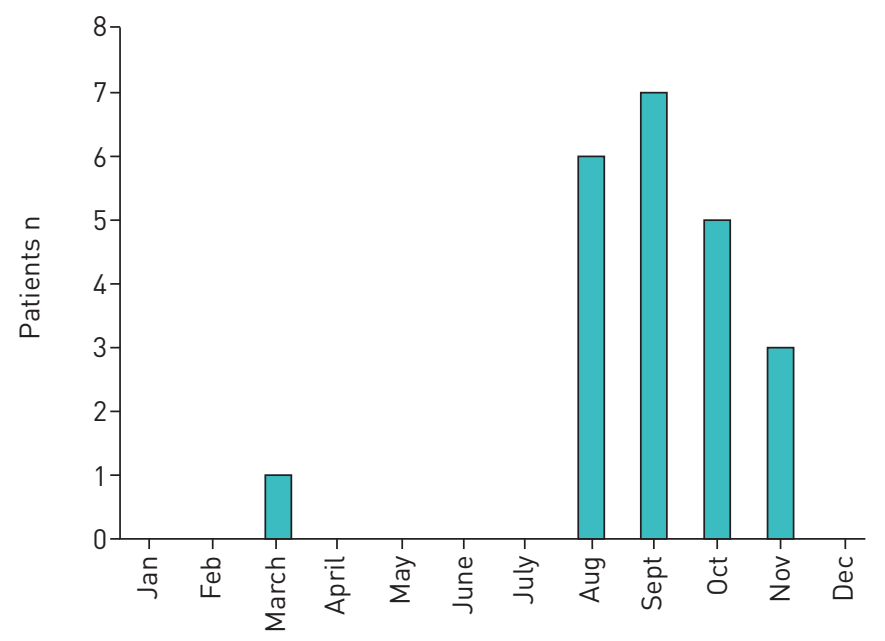




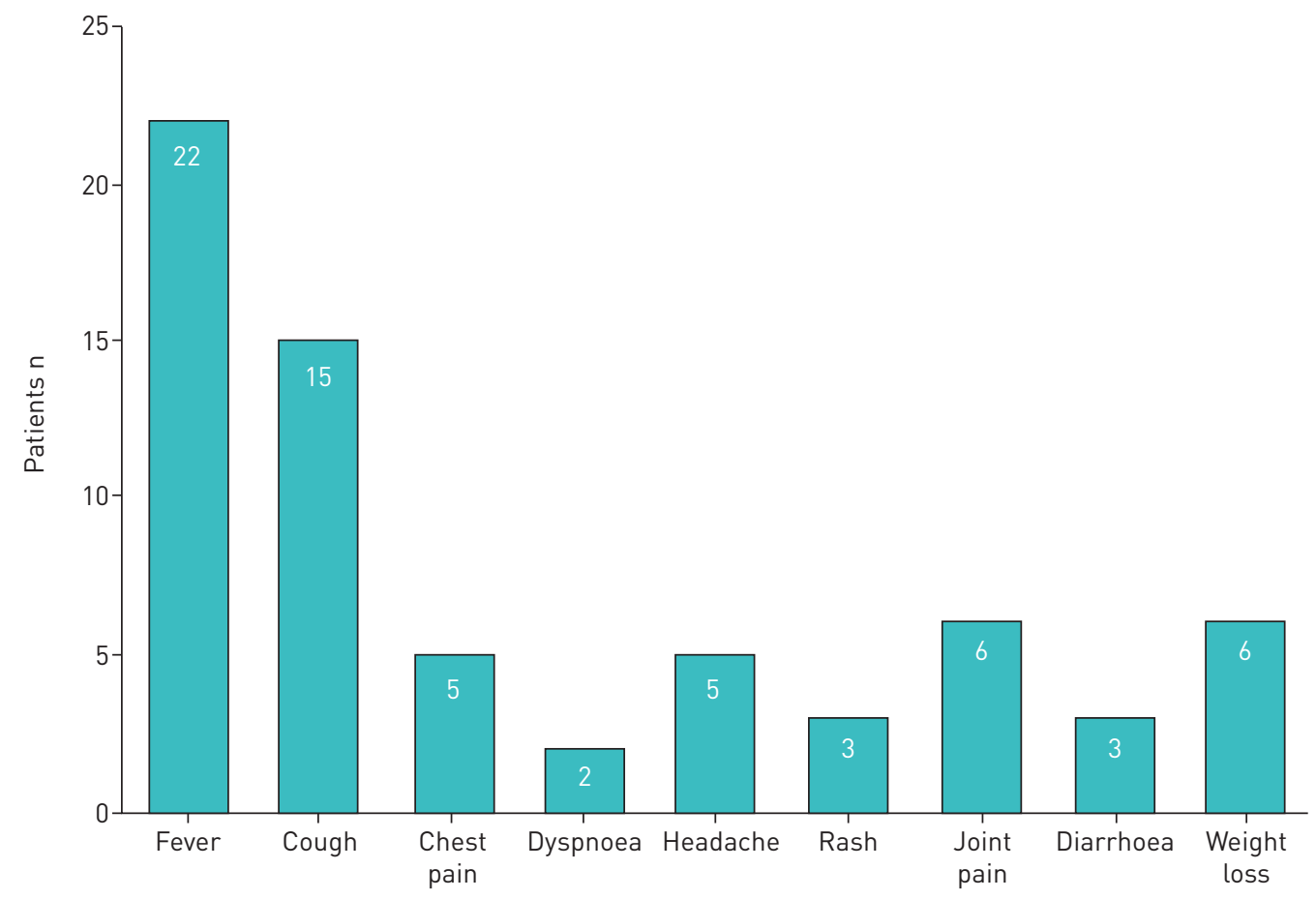

FIGURE 2 The symptoms reported by 22 patients diagnosed with primary pulmonary tularaemia. All patients had several symptoms.

\section{Radiology}

The most common findings on chest CT were enlarged mediastinal or hilar lymph nodes and pulmonary lesions (table 5). The pulmonary lesions were typically nodular and multiple, and frequently located peripherally with a blurred delimitation. They were all solid, some with signs of necrosis, cavities or bronchogram. The lymph nodes showed sign of necrosis in most patients. Pathological findings were highly FDG-avid on PET-CT. Examples of radiological findings in pulmonary tularaemia are presented in figure 3. Either the chest CT, PET-CT, or both, were described as suspicious of malignancy in 18 patients, resulting in 15 patients being investigated as possible malignancies in fast-track cancer programs. These represented $2 \%$ of the 861 patients investigated in fast-track lung cancer programs during this period in Eastern Norway.

\section{Histology/cytology}

Histological and cytological samples were retrieved from 10 patients with 17 samples in total (five EBUS with transbronchial needle aspiration (TBNA)), two bronchoscopic fine-needle aspirations (FNAs), five

TABLE 3 Results of microbiological analyses (serology, PCR and culture) in 22 patients diagnosed with primary pulmonary tularaemia at Innlandet Hospital Trust in Norway in $2016-2018$

Positive

Negative

First serological test

Second serological test

PCR EBUS-TBNA

PCR BAL

PCR lung biopsy

PCR pleural fluid

Culture"

17
5
2
1
1
0
0

0

3

0

1

11

EBUS-TBNA: endobronchial ultrasound-guided transbronchial needle aspiration; BAL: bronchoalveolar lavage. " : four out of five lung biopsies were not investigated for Francisella tularensis; ": cultures from five EBUS-TBNAs, four BALs, one pleural fluid and one computed tomography-guided lung biopsy. 
TABLE 4 Performed investigations in 22 patients diagnosed with primary pulmonary tularaemia at Innlandet Hospital Trust in Norway in 2016-2018

$\begin{array}{lr}\text { Chest CT } & 22 \\ \text { FDG-PET } & 10 \\ \text { Included in fast-track cancer programmes } & 15 \\ \text { Underwent EBUS } & 5 \\ \text { CT-guided lung biopsy } & 5 \\ \text { Bronchoscopy } & 15 \\ \text { Drainage of pleural effusion } & 1\end{array}$

CT: computed tomography; FDG-PET: fluorodeoxyglucose positron emission tomography; EBUS: endobronchial ultrasound. " : one patient (of the five) underwent CT-guided lung biopsy twice.

CT-guided lung biopsies and five bronchial mucosal biopsies). Cytological samples from one EBUS-TBNA and the two FNAs were not representative. Cytopathological examination revealed inflammatory changes in 12 samples, six with necrosis, five with granulomas and three with both granulomas and necrosis.

\section{Follow-up}

All patients underwent follow-up CT scans and/or chest radiography with a median follow-up time of 3.7 months (range 3 weeks to 7.5 months). Although all patients demonstrated regression of the radiological findings on follow-up exams, a follow-up period of 12-18 months is planned for two of the patients (one patient previously treated for breast cancer, and one patient where the biopsy initially was interpreted as neoplastic before the pathologists concluded that the most likely diagnosis was inflammation).

\section{Discussion}

The diagnosis of pulmonary tularaemia can be challenging. In the present study, the majority of the 22 patients diagnosed with pulmonary tularaemia were initially investigated in fast-track cancer programs based on suspect radiological findings. However, all of them had symptoms consistent with an infection. This study may indicate that pulmonary tularaemia is more frequent than previously reported, highlighting the importance of being aware of pulmonary tularaemia as a differential diagnosis to lung cancer in endemic areas.

\section{Epidemiology and risk exposure}

In this study, 22 patients were diagnosed with primary pulmonary tularaemia in Eastern Norway within the time course of 28 months, with a peak incidence of 3.4 in 100000 in 2017. These 22 patients

TABLE 5 Radiological findings on chest computed tomography (CT) and positron emission tomography (PET)-CT in 22 patients diagnosed with primary pulmonary tularaemia at

Innlandet Hospital Trust in Norway in 2016-2018

$\begin{array}{lc}\text { Enlarged lymph nodes }^{\#} & 18 / 22 \\ \text { Signs of necrosis in lymph nodes } & 11 / 18 \\ \text { Pulmonary lesions } & 19 / 22 \\ \text { Pulmonary noduleף } & 16 / 19 \\ \text { Pulmonary mass }{ }^{+} & 3 / 19 \\ \text { Multiple } & 12 / 19 \\ \text { Peripheral } & 16 / 19 \\ \text { Solid } & 19 / 19 \\ \text { Solid with bronchogram } & 6 / 19 \\ \text { Solid with sign of necrosis } & 4 / 19 \\ \text { Pulmonary cavities } & 2 / 19 \\ \text { Enlarged lymph nodes and pulmonary lesions } & 15 / 22 \\ \text { Pleural effusion } & 8 / 22 \\ \text { Pleural effusion and pulmonary lesions } & 5 / 22 \\ \text { Chest CT described as suspicious of malignancy } & 17 / 22 \\ \text { PET-CT described as suspicious of malignancy } & 6 / 10\end{array}$

Data are presented as $\mathrm{n} / \mathrm{N}$. The most common findings were enlarged lymph nodes and solid, nodular pulmonary lesions. ${ }^{\#}$ : $>1 \mathrm{~cm}$ in short-axis diameter; ${ }^{\text {I }}:<3 \mathrm{~cm}$ in diameter; ${ }^{+}:>3 \mathrm{~cm}$ in diameter. 

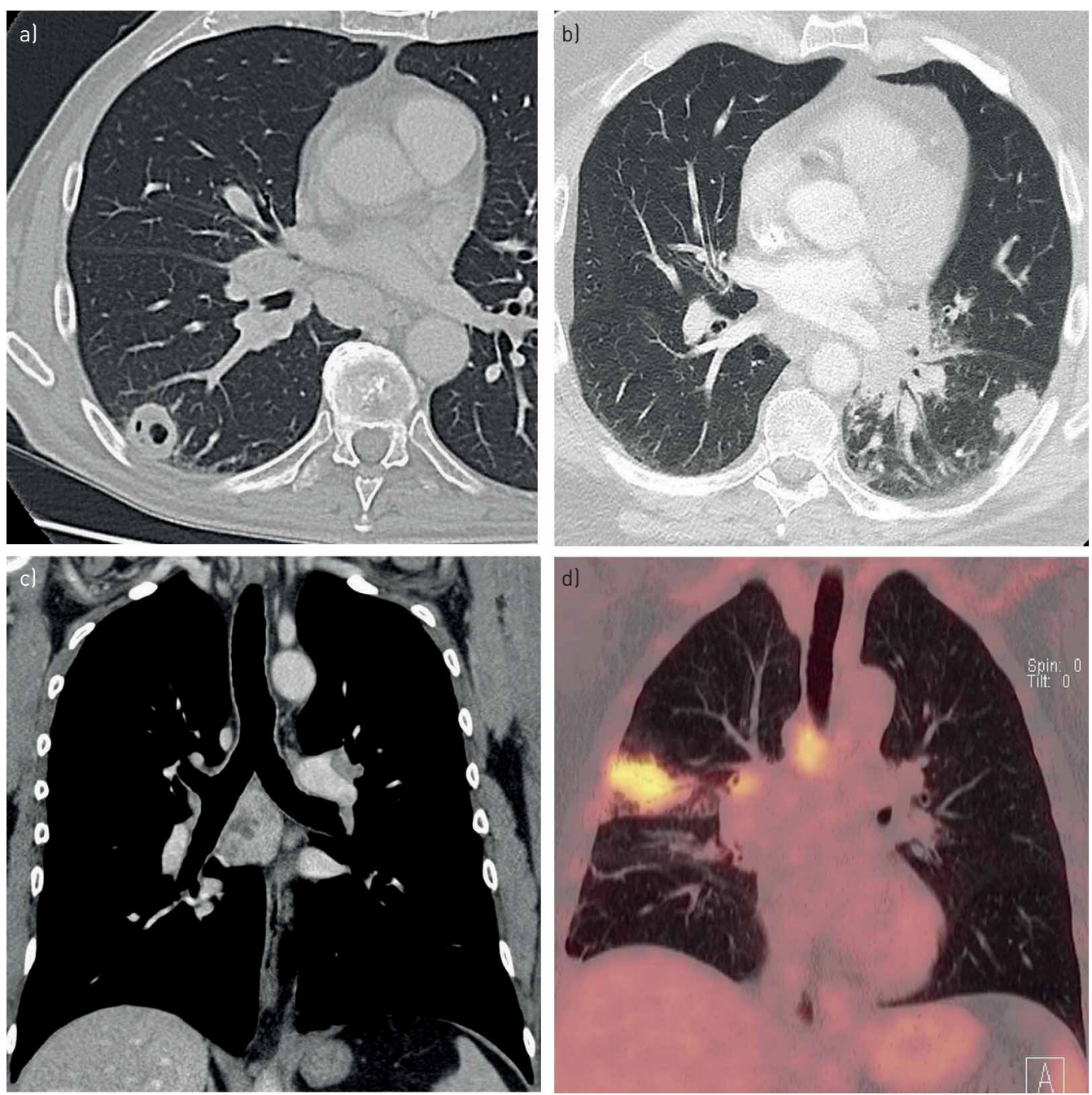

FIGURE 3 a and b) Axial computed tomography (CT) image, c) coronal CT image and d) coronal positron emission tomography-CT with examples of radiological findings in pulmonary tularaemia: al Cavity. b) Solid, nodular pulmonary lesion. c) Enlarged lymph nodes with sign of necrosis. d) Mediastinal and hilar lymph nodes and pulmonary lesion highly fluorodeoxyglucose avid.

represented $48 \%$ of all reported cases of tularaemia in the region in 2016-2018, being considerably higher than previous reports. During an outbreak in Norway in 2011, pulmonary tularaemia constituted only $13 \%$ of tularaemia cases [9]. European studies have found pulmonary tularaemia in only $3-10 \%$ of cases, but rare outbreaks have been reported [11-13, 18-20]. Although cases of tularaemia are mandatory to notify, there are no updated Norwegian statistics on the incidence of the various clinical manifestations of tularaemia.

Tularaemia has often been more frequent in Norway in years with an increase in rodent population $[8,9$, 21]. A large number of rodents were observed in Eastern Norway during 2017, possibly contributing to the peak incidence of pulmonary tularaemia [22]. In addition, a higher awareness among the pulmonologists in our region likely contributed to an increase in the diagnosis of pulmonary tularaemia. In our study, most of the patients reported risk exposure to tularaemia such as farming, hunting, wood chopping, carpentry/house rehabilitation and outdoor activities, consistent with previous reports $[3,10,12,23-25]$. We assume that transmission through wood chopping and house rehabilitation is due to aerosolisation of rodent excrements on the woodpiles or in the walls of old houses.

\section{Clinical course}

Respiratory symptoms are quite common in pulmonary tularaemia in combination with symptoms consistent with infection $[3,4,13]$. In the present study, all patients experienced fever and elevated CRP. The majority reported cough, chest pain or dyspnoea. These symptoms are however unspecific and are 
frequent in patients with lung cancer [26-28]. We assume that similar symptoms in combination with a high focus on fast-track investigations resulted in investigation in fast-track programs due to the radiology reports, even though most of the patients were referred with symptoms of infection.

Previous studies on tularaemia have reported serum CRP as moderately elevated [19, 29]. In this study, a markedly elevated CRP at a median of $155 \mathrm{mg} \cdot \mathrm{L}^{-1}$ was found. A possible explanation for this discrepancy may be that all the present patients were referred to secondary care, and thus probably were suffering from a more advanced disease. Furthermore, we collected data from patient records in primary care and thereby from a larger proportion of the illness period. It is worth noting that almost half of the patients were in spontaneous clinical recovery at the time of diagnosis, probably because of the prolonged period from referral to diagnosis in secondary care.

\section{Radiological findings}

In our experience, pulmonary tularaemia may be a radiological differential diagnosis to lung cancer. The majority of the patients had intrathoracic lymph adenopathy, approximately half with signs of necrosis, or pulmonary lesions. These lesions were solid, typically nodular, peripheral and multiple with a blurred delimitation. Due to radiological sign of malignancy [30,31], the chest CT scan was described as suspicious of malignancy in $77 \%$ of the patients, possibly contributed by a limited experience on radiological findings in pulmonary tularaemia. Consequently, the majority of the patients were primarily investigated for lung cancer in fast-track programs, despite all of them having symptoms consistent with infection and elevated CRP. Case reports have discussed the differential diagnostic challenge towards lung cancer [23, 32-34]. As these 22 patients only represented $2 \%$ of all the patients investigated in fast-track lung cancer program in the region, our findings highlight the need of detailed patient interviews in endemic regions, focusing on symptoms and risk of tularaemia.

Since 2014, cancer pathways for fast-track diagnosis and treatment have been mandatory in Norway [35], and in our opinion, probably influenced the clinicians' ordering of supplementary diagnostic investigations. As part of the program, 10 patients underwent an FDG-PET scan. In support of previous case series [23, 32], the PET-CT scan showed highly FDG-avid lymph nodes and lesions, considered indistinguishable from malignancy in six cases (60\%). However, in a recent study from Finland, malignancy was the first suspected diagnosis in only $3 \%$ of the pulmonary tularaemia cases [13]. This discrepancy could be due to a higher incidence and thereby a higher awareness, in combination with lack of mandatory cancer programs in Finland [36].

\section{Investigations}

EBUS-TBNA is a well-established method to examine mediastinal and hilar lymph nodes. Approximately half the patients in this study were referred to EBUS-TBNA, on suspicion of malignancy. EBUS was performed on five patients, and four patients underwent a CT-guided lung biopsy for the same reason. In hindsight, it would have been prudent to wait for results of the serological tests.

To the best of our knowledge, only two papers have previously reported on the endobronchial findings in pulmonary tularaemia $[25,32]$. The findings in the present study were highly variable from normal to widespread inflammation, and even suspicious of cancer in one patient who developed suppuration of enlarged hilar lymph nodes. Cytopathological samples are not diagnostic for tularaemia, typically showing unspecific inflammation with or without necrosis and granulomas [23, 25, 37-39]. Based on our experience, bronchoscopy and EBUS-TBNA are likely unhelpful in the diagnosis of pulmonary tularaemia.

\section{The diagnosis}

Because of nonspecific symptoms and radiological findings, the diagnosis of pulmonary tularaemia can be challenging if not suspected at an early stage, illustrated in a case example in the supplementary material. The differential diagnostic challenge towards lung cancer may result in lengthy and comprehensive investigations [23, 32-34]. In our study, it took a median of 17.5 days (range 7-75 days) from referral to diagnosis in secondary care with 10 patients receiving a confirmed diagnosis after $>3$ weeks. The time delay from onset of symptoms until diagnosis correlates with the prevalence of tularaemia [3, 12, 18]. Given that the incidence in Norway is among the highest in Europe, the diagnostic delay in our study seems somewhat disappointing.

In our experience, it is important to consider pulmonary tularaemia as a differential diagnosis at an early stage, especially if the patient is living in or has recently visited rural areas in an endemic region. Serology should be ordered on clinical suspicion, not as a standard test in patients with respiratory infections. A positive serological test is usually diagnostic and represents an easy way to confirm tularaemia, and can prevent unnecessary and expensive investigations such as PET-CT, EBUS-TBNA and CT-guided lung biopsies, with their inherent risk of complications and patient distress. 
Serological tests are defined as the gold standard in the diagnosis of tularaemia. It is essential to recognise that the antibody response to tularaemia is delayed, often until 2-4 week after onset of disease, as observed in five of the patients with an initial negative serological result [3, 40]. We recommend repeating the serological test if faced with a negative initial test in a patient suspected of having pulmonary tularaemia. The sensitivity of PCR in pulmonary specimens is unknown, but probably far lower than serology. However, unlike the antibody response, PCR can be detected early in the course [40]. In our opinion, and supported by the WHO guidelines, pulmonary tularaemia can confidently be diagnosed based on positive serology in a patient with clinical and radiological findings compatible with pulmonary tularaemia.

\section{Strengths and limitations}

Although this study on primary pulmonary tularaemia is one of the largest in the last decades, only 22 patients were included. Patients with a less severe or shorter course of illness will most likely not be hospitalised or referred to radiological investigations. We thus assume that the patients in this study might be just the tip of the iceberg. Considering the challenging diagnosis of pulmonary tularaemia, it is highly questionable as to whether the diagnosis can be made on pure clinical grounds in primary care. We did not have access to individual patient data from the Norwegian Surveillance System for Communicable Diseases registry, and if there were any patients diagnosed and treated exclusively by their primary care physician, they were not included.

Future studies should aim to enrol a larger population and investigate in further detail the differing risk factors, symptoms and investigative results in pulmonary tularaemia and lung cancer. In the last decades, a multitude of new technological investigations have become available, such as CT, PET-CT and EBUS. To the best of our knowledge, the present study is the first to focus on radiological findings on chest CT and PET-CT in pulmonary tularaemia within the mindset of cancer investigation. Studies with larger populations are needed to describe the diversity of radiological pathology in pulmonary tularaemia.

\section{Conclusion}

Pulmonary manifestations in tularaemia are possibly more frequent than previously reported. Our most remarkable finding is that the majority of patients were initially investigated as suspicious of lung cancer based on the radiology reports. Pulmonary tularaemia may be an important differential diagnosis to lung cancer in endemic areas.

Acknowledgements: The authors would like to thank Astrid Sandnes (Innlandet Hospital Trust, Gjøvik, Norway), Peter Jebsen (Oslo University Hospital (Rikshospitalet), Oslo, Norway) and Kåre Bergh (Trondheim University Hospital, Trondheim, Norway) for their assistance with preparation of the manuscript. This work was previously presented as a poster at the European Respiratory Society International Congress in Paris, France, in 2018.

Conflict of interest: None declared.

\section{References}

1 Carvalho CL, Lopes de Carvalho I, Zé-Zé L, et al. Tularaemia: a challenging zoonosis. Comp Immunol Microbiol Infect Dis 2014; 37: 85-96.

2 Maurin M, Gyuranecz M. Tularaemia: clinical aspects in Europe. Lancet Infect Dis 2016; 16: 113-124.

3 Tarnvik A, Berglund L. Tularaemia. Eur Respir J 2003; 21: 361-373.

4 World Health Organization. WHO guidelines on tularemia. 2007. www.who.int/csr/resources/publications/WHO_ CDS_EPR_2007_7.pdf?ua=1 Date last updated: July 2007. Date last accessed: 18 February 2019.

5 Hestvik G, Warns-Petit E, Smith LA, et al. The status of tularemia in Europe in a one-health context: a review. Epidemiol Infect 2015; 143: 2137-2160.

6 Eden JS, Rose K, Ng J, et al. Francisella tularensis ssp. holarctica in ringtail possums, Australia. Emerg Infect Dis 2017; 23: 1198-1201.

7 Jackson J, McGregor A, Cooley L, et al. Francisella tularensis subspecies holarctica, Tasmania, Australia, 2011. Emerg Infect Dis 2012; 18: 1484-1486.

8 The_Norwegian_Institute_of_Public_Health. Smittevernveilederen. 2010. www.fhi.no/nettpub/ smittevernveilederen/sykdommer-a-a/tularemi-veileder-for-helseperson/ Date last updated: 2 February, 2018. Date last accessed: 16 December, 2018.

9 Larssen KW, Bergh K, Heier BT, et al. All-time high tularaemia incidence in Norway in 2011: report from the national surveillance. Eur J Clin Microbiol Infect Dis 2014; 33: 1919-1926.

10 Feldman KA, Enscore RE, Lathrop SL, et al. An outbreak of primary pneumonic tularemia on Martha's Vineyard. N Engl J Med 2001; 345: 1601-1606.

11 Maurin M, Pelloux I, Brion JP, et al. Human tularemia in France, 2006-2010. Clin Infect Dis 2011; 53: e133-e141.

12 Mailles A, Vaillant V. 10 years of surveillance of human tularaemia in France. Euro Surveill 2014; 19: 20956.

13 Vayrynen SA, Saarela E, Henry J, et al. Pneumonic tularaemia: experience of 58 cases from 2000 to 2012 in Northern Finland. Infect Dis 2017; 49: 758-764.

14 Rubin SA. Radiographic spectrum of pleuropulmonary tularemia. AJR Am J Roentgenol 1978; 131: 277-281.

15 Miller RP, Bates JH. Pleuropulmonary tularemia. A review of 29 patients. Am Rev Respir Dis 1969; 99: 31-41.

16 Syrjälä H, Kujala P, Myllylä V, et al. Airborne transmission of tularemia in farmers. Scand J Infect Dis 1985; 17: 371-375. 
17 Sjöstedt A, Eriksson U, Berglund L, et al. Detection of Francisella tularensis in ulcers of patients with tularemia by PCR. J Clin Microbiol 1997; 35: 1045-1048.

18 Pérez-Castrillón JL, Bachiller-Luque P, Martín-Luquero M, et al. Tularemia epidemic in northwestern Spain: clinical description and therapeutic response. Clin Infect Dis 2001; 33: 573-576.

19 Eliasson H, Back E. Tularaemia in an emergent area in Sweden: an analysis of 234 cases in five years. Scand J Infect Dis 2007; 39: 880-889.

20 Siret V, Barataud D, Prat M, et al. An outbreak of airborne tularaemia in France, August 2004. Euro Surveill 2006; 11: 58-60.

21 Brantsaeter AB, Krogh T, Radtke A, et al. Tularaemia outbreak in northern Norway. Euro Surveill 2007; 12 E070329.2.

22 The Norwegian Veterinary Institute. Press release. www.vetinst.no/nyheter/mange-tilfelle-av-harepest-i-sor-noreg Date last updated: 16 October, 2017. Date last accessed: 17, December, 2017.

23 Hillerdal G, Kolbeck K, Jacobsson H. Pulmonary tularemia: rare disease that can be interpreted as lung cancer. Serology provides the correct diagnosis, as shown by case reports. Lakartidningen 2013; 110: 1280-1281.

24 Valipour A, Koller H, Kreuzer A, et al. A case of primary tularemic pneumonia presenting with necrotizing mediastinal and hilar lymph nodes. Wien Klin Wochenschr 2003; 115: 196-199.

25 Syrjala H, Sutinen S, Jokinen K, et al. Bronchial changes in airborne tularemia. J Laryngol Otol 1986; 100: $1169-1176$.

26 Kocher F, Hilbe W, Seeber A, et al. Longitudinal analysis of 2293 NSCLC patients: a comprehensive study from the TYROL registry. Lung Cancer 2015; 87: 193-200.

27 Tulek B, Koylu H, Kanat F, et al. Serum C-reactive protein and procalcitonin levels in non-small cell lung cancer patients. Contemp Oncol (Pozn) 2013; 17: 68-72.

28 Hyde L, Hyde CI. Clinical manifestations of lung cancer. Chest 1974; 65: 299-306.

29 Syrjälä H. Peripheral blood leukocyte counts, erythrocyte sedimentation rate and C-reactive protein in tularaemia caused by the type B strain of Francisella tularensis. Infection 1986; 14: 51-54.

30 Hollings N, Shaw P. Diagnostic imaging of lung cancer. Eur Respir J 2002; 19: 722-742.

31 MacMahon H, Naidich DP, Goo JM, et al. Guidelines for management of incidental pulmonary nodules detected on CT images: from the Fleischner Society 2017. Radiology 2017; 284: 228-243.

32 Fachinger P, Tini GM, Grobholz R, et al. Pulmonary tularaemia: all that looks like cancer is not necessarily cancer - case report of four consecutive cases. BMC Pulm Med 2015; 15: 27.

33 Franzen D, Muller F, Bode PK. Lung mass and tularaemia. OJM 2016; 109: 417-418.

34 Odegaard K, Boersma B, Keegan J. Atypical presentations of tularemia. S D Med 2017; 70: 207-209.

35 The Norwegian Directorate of Health. Pakkeforløp kreft. 2017. https://helsedirektoratet.no/kreft/pakkeforlop-forkreft Date last updated: 24 March, 2017. Date last accessed: 18 January, 2018.

36 Christensen NL, Jekunen A, Heinonen S, et al. Lung cancer guidelines in Sweden, Denmark, Norway and Finland: a comparison. Acta Oncol 2017; 56: 943-948.

37 Navarro P, Garcia-Moliner ML, McMahon JH, et al. Histologic, immunohistochemical, microbiological, molecular biological and ultrastructural characterization of pulmonary tularemia. Pathol Res Pract 2011; 207: 63-66.

38 Markoc F, Koseoglu RD, Koc S, et al. Tularemia in differential diagnosis of cervical lymphadenopathy: cytologic features of tularemia lymphadenitis. Acta Cytol 2014; 58: 23-28.

39 Tuncer E, Onal B, Simsek G, et al. Tularemia: potential role of cytopathology in differential diagnosis of cervical lymphadenitis: multicenter experience in 53 cases and literature review. APMIS 2014; 122: 236-242.

40 Tarnvik A, Chu MC. New approaches to diagnosis and therapy of tularemia. Ann NY Acad Sci 2007; 1105: 378-404. 